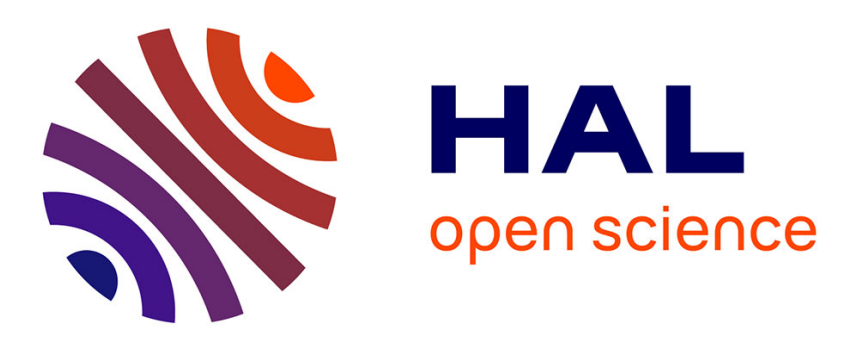

\title{
A Geometric Point of View on Parallel Interconnection of Buck Converters
}

Jérémie Kreiss, Jean-François Trégouët, Romain Delpoux, Jean-Yves

Gauthier, Xuefang Lin-Shi

\section{- To cite this version:}

Jérémie Kreiss, Jean-François Trégouët, Romain Delpoux, Jean-Yves Gauthier, Xuefang Lin-Shi. A Geometric Point of View on Parallel Interconnection of Buck Converters. ECC, Jun 2018, Limassol, Cyprus. pp.70-75, 10.23919/ECC.2018.8550575 . hal-02053176

\section{HAL Id: hal-02053176 https://hal.science/hal-02053176}

Submitted on 1 Mar 2019

HAL is a multi-disciplinary open access archive for the deposit and dissemination of scientific research documents, whether they are published or not. The documents may come from teaching and research institutions in France or abroad, or from public or private research centers.
L'archive ouverte pluridisciplinaire HAL, est destinée au dépôt et à la diffusion de documents scientifiques de niveau recherche, publiés ou non, émanant des établissements d'enseignement et de recherche français ou étrangers, des laboratoires publics ou privés. 


\title{
A Geometric Point of View on Parallel Interconnection of Buck Converters
}

\author{
Jérémie Kreiss ${ }^{1}$, Jean-François Trégouët ${ }^{1}$, Romain Delpoux ${ }^{1}$, Jean-Yves Gauthier ${ }^{1}$ and Xuefang Lin-Shi ${ }^{1}$
}

\begin{abstract}
This paper tackles the current sharing problem for interconnected power converters. Specifically, it considers a single load, fed by buck converters via a common DC bus. In such a case, it has been recently shown that dynamics related to (i) voltage regulation and (ii) current distribution can be completely separated without resorting to frequency separation argument, which inevitably lowers achievable performance. In this paper, the origin of this separation is investigated. A comprehensive analysis is provided by relying on geometric techniques. Controller design example exploiting the new structure is also proposed. Numerical simulations promote the decomposition benefit and illustrate the geometric notions.
\end{abstract}

\section{INTRODUCTION}

Nowadays, connecting several power converters in parallel to a single load becomes more and more common. Indeed, in many applications such as Microgrids (see [1] for example) or Low-voltage/High-current power supplies, this kind of parallel interconnection of converters is used. Despite a larger number of electrical components, this interconnection benefits from several advantages such as increased reliability, ease of maintenance and repair, improved thermal management, reduced output ripple by interleaving phase of Pulse Width Modulation (PWM), etc. Each of them being a consequence of the possibility to freely distribute the load current on each converter.

Several solutions for controlling such a system have been proposed in the literature (see e.g. [2], [3], [4]). The main challenge is to regulate output voltage as well as current distribution, corresponding to power-flow into the interconnection. Those two dynamics are coupled, though. To cope with this difficulty, most of existing solutions propose control design procedure relying on several SISO transfer functions shaping. However, deriving conditions for closedloop stability in this framework seems rather involved, so that frequency separation is often ultimately used as the key argument, which inevitably lowers achievable performance by imposing a slow current distribution dynamics as in [1].

In stark contrast with this approach, new solution has been recently proposed in [5], resorting to both state and input change of coordinates rather than frequency separation to completely separate voltage and current distribution dynamics, hence offering tractability without sacrificing performance. Arbitrary number of DC/DC buck converters having distinct characteristics was considered.

In this paper, we propose to look at main result of [5] in a different way. Specifically, by using geometric control point

\footnotetext{
${ }^{1}$ Every author is with Laboratoire Ampère, INSA Lyon, Université de Lyon, 20, Avenue Albert Einstein, 69100 Villeurbanne, France firstname. lastnamedinsa-lyon.fr
}

of view (see [6], [7], [8]), we aim to bring a fresh perspective to the origin of the change of coordinates provided by [5]. Main contribution of this paper consists in recovering basis vector of the new coordinates by exploiting properties of output nulling controlled invariant subspaces and discussing implication of this reformulation in terms of control design.

The paper is organized as follows: After defining the control problem in Section II and introducing background materials on geometric control theory in Section III, origin of the change of coordinates is described in Section IV. In Section V, benefits of this reformulation in terms of control design is discussed. In Section VI, some simulations are performed in order to illustrate the proposed approach. Finally, conclusions are offered in Section VII.

Notation: The symbol $\mathbf{I}_{m}$ stands for the identity matrix of dimensions $m \times m$. The null matrix of size $m \times n$ is denoted by $\mathbf{0}_{m \times n}$. The vector (column matrix) of size $m$ for which every entry is $1(0)$ is denoted by $\mathbf{1}_{m}\left(\mathbf{0}_{m}\right)$. The notation $x_{k}$ refers to the $k$-th element of the vector $x$, with 1 being the index of the first element. The operator "diag" builds diagonal matrix from entries of the input vector argument.

\section{Problem Statement}

We consider the electrical circuit shown in Fig. 1 which corresponds to parallel interconnection of $m$ buck converters sharing a single capacitor and connected to a common resistive load $R$. Converters are controlled via PWM where $d_{k}$ refers to duty cycle of $k$-th converter. Index $k$ belongs to the following set:

$$
\mathscr{K}:=\{1, \ldots, m\}
$$

Voltage of DC bus is denoted by $v$ and current in $k$-th inductor $L_{k}$ is referred to as $i_{k}$. We gather those state variables in the following vector:

$$
\mathbb{R}^{m+1} \ni x:=\left[i^{\top} v\right]^{\top}
$$

where $i=\left[i_{1}, \ldots, i_{m}\right]^{\top}$. Magnitude of voltage sources $E_{k}$ are supposed to be known and constant. Capacitor $C$ is connected in parallel to the load $R$.

Throughout this paper, we assume that (i) switching frequency $f_{s}$ is sufficiently large for the dynamics to be approximated by an average continuous time model, (ii) converters remain in continuous conduction mode and (iii) electrical components and switches are ideals, i.e. parasitic elements (resistances, losses) can be neglected.

Using Kirchoff's laws and considering previous assumptions, dynamics of the circuit represented by Fig. 1 is 


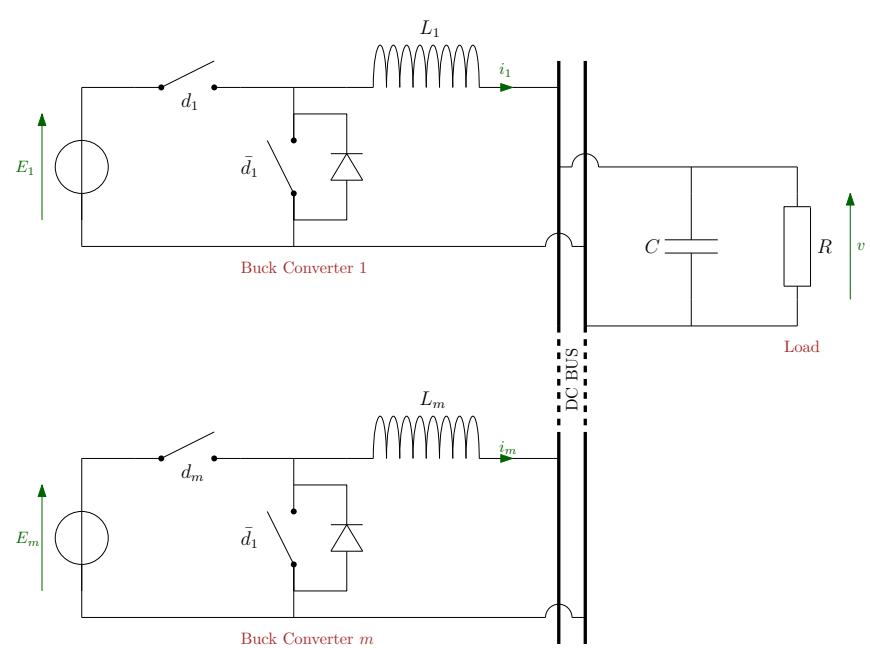

Fig. 1. Electrical schematic

expressed by

$$
\begin{aligned}
\forall k \in \mathscr{K}, L_{k} \frac{d i_{k}}{d t} & =-v+E_{k} d_{k}, \\
C \frac{d v}{d t} & =\sigma-\frac{v}{R},
\end{aligned}
$$

where

$$
\sigma:=\sum_{k \in \mathscr{K}} i_{k}=\mathbf{1}_{m}^{\top} i
$$

refers to the total current. Eq. (1a) refers to the dynamics of output current produced by each converters whereas (1b) describes the output voltage dynamics.

The main control goal on this system is to regulate the bus voltage $v$ to a given constant value $v_{r}$. This leads us to the following state-space representation:

$$
\begin{aligned}
{\left[\begin{array}{cc}
\operatorname{diag}\{L\} & \mathbf{0} \\
\mathbf{0} & C
\end{array}\right] \dot{x} } & =\left[\begin{array}{cc}
\mathbf{0} & -\mathbf{1}_{m} \\
\mathbf{1}_{m}^{\top} & -1 / R
\end{array}\right] x+\left[\begin{array}{c}
\operatorname{diag}\{E\} \\
\mathbf{0}_{m}^{\top}
\end{array}\right] d \\
z & =\left[\begin{array}{ll}
\mathbf{0}_{m}^{\top} & 1
\end{array}\right] x
\end{aligned}
$$

where $z$ represents the regulated output.

Remark (Controllability of (2)). It can be easily checked that system (2) is controllable for any $R \neq 0$. Indeed, the first $2 m-1$ columns of controllability matrix $\mathscr{C}$ reads

$$
\left[\begin{array}{cc}
\operatorname{diag}\{E\} \operatorname{diag}\{L\}^{-1} & 0 \\
0 & E^{\top} \operatorname{diag}\{L\}^{-1}
\end{array}\right] .
$$

Clearly this matrix has full row $\operatorname{rank}^{1}$ so that $\mathscr{C}$ inherits from this property.

\section{BACKGROUND ON GEOMETRIC CONTROL THEORY}

In the considered problem, $m$ independent control $d_{k}$ are used to impose asymptotic value of scalar signal $z=v$. This suggests that degrees of freedom exist in the way this goal is achieved. Indeed, (1b) shows that $v$ depends on the total current $\sigma$ instead of each $i_{k}$ individually. As a result, the way

\footnotetext{
${ }^{1}$ For the problem to be meaningful, it holds $E_{k}>0$ and $L_{k}>0$ for all $k \in \mathscr{K}$.
}

$\sigma$ is distributed among converters may not impact $z$. This intuition was the core of the reasoning behind the derivation of the change of coordinates provided in [5].

In this paper, we use a different path to recover main result of [5] and, thereby, provides deeper investigation on the considered problem. Specifically, observe that the previous remark is equivalent to saying that only a projection of the state vector, that is $\left[\mathbf{1}_{m}^{\top}, 0\right] x$ and $\left[\mathbf{0}_{m}^{\top}, 1\right] x$, affects the regulated output $z$. To properly isolate part of the state space which does not impact $z$, we refer to some geometric tools (see [6], [7] and [8]) which are now introduced.

\section{A. Output nulling controlled invariant subspaces}

Consider the following LTI system:

$$
\Sigma:\left\{\begin{array}{l}
\dot{x}=A x+B u \\
z=C x+D u
\end{array}\right.
$$

with state $x \in \mathbb{R}^{n}$, control input $u \in \mathbb{R}^{m}$ and regulated output $z \in \mathbb{R}^{p}$. We denote by $\mathscr{V}^{*}$ the largest output-nulling controlled invariant subspace of $\Sigma$, i.e., the largest subspace $\mathscr{V}$ of $\mathbb{R}^{n}$ such that (see $[6, \mathrm{Ch} .5]$ )

$$
\left[\begin{array}{l}
A \\
C
\end{array}\right] \mathscr{V} \subset(\mathscr{V} \times \mathbf{0})+\operatorname{Im}\left\{\left[\begin{array}{l}
B \\
D
\end{array}\right]\right\}
$$

or, equivalently, the largest subspace $\mathscr{V}$ of $\mathbb{R}^{n}$ for which a matrix $F \in \mathbb{R}^{m \times n}$ exists such that

$$
(A+B F) \mathscr{V} \subset \mathscr{V} \subset \operatorname{Ker}\{C+D F\}
$$

It follows immediately from (3), that $\mathscr{V}^{*}$ has the properties below:

1) "controlled invariance": From the first inclusion of (3), it follows that every solution of $\dot{x}=(A+B F) x$ that starts in $\mathscr{V}^{*}$ remains in $\mathscr{V}^{*}$ for all $t \geq 0$;

2) "output nulling": From the second inclusion of (3), it comes out that every element $x \in \mathscr{V}^{*}$ ensure that $z=$ $(C+D F) x$ is identically zero.

Any matrix $F$ satisfying (3) is called a friend of $\mathscr{V}$. When state-feedback $u=F x$ with such a matrix is implemented, resulting closed-loop system induces $z(t)=\mathbf{0},(t \geq 0)$ for all $x(0) \in \mathscr{V}^{\star}$.

Let $\mathscr{R}^{*}$ be the so-called largest reachability subspace of $\mathscr{V}^{*}$. When a LTI system contains some uncontrollable zeros, $\mathscr{R}^{*}$ corresponds to the controllable part of $\mathscr{V}^{*}$.

\section{B. A matrix view point}

Assume that there is no uncontrollable invariant zeros so that $\mathscr{R}^{*}=\mathscr{V}^{*}$. For the sake of simplicity, we also consider that $D=0 .^{2}$ Let $\rho:=\operatorname{dim} \mathscr{R}^{*}$. Choose $T \in \mathbb{R}^{n \times n}$ as an invertible matrix such that its $\rho$ first columns span $\mathscr{R}^{*}$. Choose $G=\left[\begin{array}{ll}G_{\alpha} & G_{\beta}\end{array}\right] \in \mathbb{R}^{m \times m}$ as an invertible matrix such that $\operatorname{Im}\left\{B G_{\alpha}\right\}=\mathscr{R}^{*}$. Choose finally $F \in \mathbb{R}^{m \times n}$ as a friend of $\mathscr{R}^{*}$. Apply the regular feedback transformation $u=F x+G \tilde{u}$ and the coordinate change $\xi=T^{-1} x$ to the system $\Sigma$ in order to obtain

$$
\dot{\xi}=\bar{A}_{F} \xi+\bar{B} \tilde{u}, \quad z=\bar{C} \xi
$$

${ }^{2}$ Those two assumptions are valid for system (2). 
where $\bar{A}_{F}=T^{-1}(A+B F) T, \bar{B}=T^{-1} B G$ and $\bar{C}=C T$. According to [9], system (4) have the following structure

$$
\left[\begin{array}{c|c}
\bar{A}_{F} & \bar{B} \\
\hline \bar{C} & 0
\end{array}\right]=\left[\begin{array}{cc|cc}
\bar{A}_{11} & \bar{A}_{12} & \bar{B}_{11} & \bar{B}_{12} \\
0 & \bar{A}_{22} & 0 & \bar{B}_{22} \\
\hline 0 & \bar{C}_{12} & 0 & 0
\end{array}\right]
$$

where $\bar{A}_{11} \in \mathbb{R}^{\rho \times \rho}, \bar{A}_{22} \in \mathbb{R}^{(n-\rho) \times(n-\rho)}$ and $\bar{B}_{11} \in \mathbb{R}^{\rho \times \rho_{1}}$ with $\rho_{1}$ being the number of columns of $G_{\alpha}$ or equivalently the size of the input subspace for which $B u \in \mathscr{R}^{*}$.

The upper triangular structure of (5) allows for a decomposition into two subsystems. Let $\eta \in \mathbb{R}^{\rho}$ and $\varphi \in \mathbb{R}^{n-\rho}$ be parts of the state vector in the new coordinates such that: $\xi=\left[\begin{array}{ll}\eta^{\top} & \varphi^{\top}\end{array}\right]^{\top}$. Similarly let $\tilde{u}_{\alpha} \in \mathbb{R}^{\rho_{1}}$ and $\tilde{u}_{\beta} \in \mathbb{R}^{m-\rho_{1}}$ such that $\tilde{u}=\left[\begin{array}{ll}\tilde{u}_{\alpha}^{\top} & \tilde{u}_{\beta}^{\top}\end{array}\right]^{\top}$. We can write dynamics of (4) as follows

$$
\begin{aligned}
\dot{\eta} & =\bar{A}_{11} \eta+\bar{B}_{11} \tilde{u}_{\alpha}+\bar{A}_{12} \varphi+\bar{B}_{12} \tilde{u}_{\beta}, \\
\dot{\varphi} & =\bar{A}_{22} \varphi+\bar{B}_{22} \tilde{u}_{\beta}, \\
z & =\bar{C}_{12} \varphi .
\end{aligned}
$$

Relation (6b) points out that the dynamics of $\varphi$ is independent of both $\eta$ and $\tilde{u}_{\alpha}$. From (6c), the output $z$ inherits from the property. Trajectories of $\varphi$ are usually denoted as the "external dynamic" whereas "internal dynamics" refers to trajectories of $\eta$ (see [8]). $\eta$ is the part of the state for which the output vanishes identically to zero. On (6a), we notice that $\bar{A}_{12}$ and $\bar{B}_{12}$ matrices represent an unilateral coupling from the external dynamic and input to the internal dynamic. This discussion is summarized in Fig. 2.

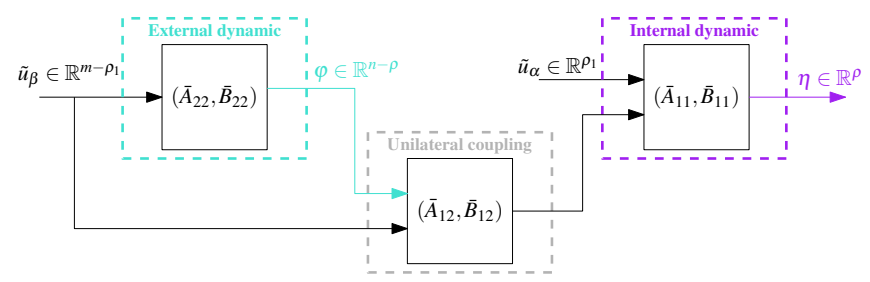

Fig. 2. New cascaded open loop model

\section{HOW TO SEPARATE VOLTAGE REGULATION FROM CURRENT DISTRIBUTION?}

The goal of the paper is to provide an in-depth analysis of the main result of [5]: There exists a suitable change of variable on both state $(i, v)$ and input $d$ that completely decouples voltage dynamics from current distribution trajectories. This section makes use of material of the previous section in order to unveil how geometry of the considered problem provides such a change of coordinates.

\section{A. Main results of [5]}

Let us select the new state variables $(\delta, \sigma, v) \in \mathbb{R}^{m-1} \times$ $\mathbb{R} \times \mathbb{R}$ via

$$
\left[\begin{array}{l}
\delta \\
\sigma \\
v
\end{array}\right]=T^{-1}\left[\begin{array}{l}
i \\
v
\end{array}\right]
$$

with

$$
T^{-1}:=\left[\begin{array}{cc}
\Gamma_{m}^{\top} & \mathbf{0}_{m-1} \\
\mathbf{1}_{m}^{\top} & 0 \\
\mathbf{0}_{m}^{\top} & 1
\end{array}\right] \in \mathbb{R}^{(m+1) \times(m+1)}
$$

and where $\Gamma_{m} \in \mathbb{R}^{m \times(m-1)}$ is defined as follows

$$
\Gamma_{m}:=\left[\begin{array}{cccc}
1 & 0 & \cdots & 0 \\
-1 & 1 & \ddots & \vdots \\
0 & -1 & \ddots & 0 \\
\vdots & \ddots & \ddots & 1 \\
0 & \cdots & 0 & -1
\end{array}\right]=\left[\begin{array}{c}
I_{m-1} \\
\mathbf{0}_{m-1}^{\top}
\end{array}\right]-\left[\begin{array}{l}
\mathbf{0}_{m-1}^{\top} \\
I_{m-1}
\end{array}\right] .
$$

Note that $T$ is indeed invertible and it can be easily verified that this matrix reads

$$
T=\left[\begin{array}{ccc}
\Gamma_{m}\left(\Gamma_{m}^{\top} \Gamma_{m}\right)^{-1} & \frac{1}{m} \mathbf{1}_{m} & \mathbf{0}_{m} \\
\mathbf{0}_{m-1}^{\top} & 0 & 1
\end{array}\right] .
$$

Remark (Physical meaning of $\delta$ ). It is worth mentioning that the new state variable $\sigma$, the total current, appears explicitly. In addition to that, remark that new coordinates $\delta$ admits a physical interpretation as it holds

$$
\delta=\left[\left(i_{1}-i_{2}\right),\left(i_{2}-i_{3}\right), \ldots,\left(i_{m-1}-i_{m}\right)\right]^{\top}
$$

so that this vector reflects the current distribution.

Regarding the input, [5] introduces the following change of coordinates

$$
\begin{aligned}
& d=\operatorname{diag}\{E\}^{-1} \operatorname{diag}\{L\}\left[\Gamma_{m}\left(\Gamma_{m}^{\top} \Gamma_{m}\right)^{-1} \quad \frac{1}{m} \mathbf{1}_{m}\right] \\
& {\left[\begin{array}{cc}
\operatorname{diag}\left\{\Delta^{*}\left(L^{-1}\right)\right\} \operatorname{diag}\left\{\Delta^{*} E\right\} & \mathbf{0} \\
\mathbf{0} & E_{\mathrm{eq}} / L_{\mathrm{eq}}
\end{array}\right]\left[\begin{array}{l}
\lambda \\
\mu
\end{array}\right]}
\end{aligned}
$$

which decomposes $d$ into $\lambda \in \mathbb{R}^{m-1}$ and $\mu \in \mathbb{R}$. The following constants have been used:

$$
\mathbb{R}^{+*} \ni 1 / L_{\mathrm{eq}}:=\sum_{k} 1 / L_{k}, \quad \mathbb{R}^{+*} \ni E_{\mathrm{eq}}:=\min _{k} E_{k},
$$

and the operator $\Delta$ reads

$$
\mathbb{R}^{p} \ni y \mapsto \Delta y:=\Gamma_{p}^{\top} y,
$$

whereas ${ }^{3} \Delta^{*}$ is such that:

$$
\mathbb{R}^{p} \ni y \mapsto\left(\Delta^{*} y\right)_{k}:= \begin{cases}(\Delta y)_{k}, & \left(y_{k} \neq y_{k+1}\right) \\ 1, & (\text { otherwise })\end{cases}
$$

Resulting dynamics of the open loop in the new coordinates is as follows:

$$
\begin{array}{r}
\frac{\mathrm{d}}{\mathrm{d} t}\left[\begin{array}{l}
\delta \\
\sigma \\
v
\end{array}\right]= \\
{\left[\begin{array}{ccc}
\mathbf{0} & \mathbf{0} & -\Delta\left(L^{-1}\right) \\
\mathbf{0} & 0 & -1 / L_{\mathrm{eq}} \\
\mathbf{0} & 1 / C & -1 /(R C)
\end{array}\right]\left[\begin{array}{l}
\delta \\
\sigma \\
v
\end{array}\right]} \\
+\left[\begin{array}{cc}
E_{\delta} L_{\delta}^{-1} & \mathbf{0} \\
\mathbf{0} & E_{\mathrm{eq}} / L_{\mathrm{eq}} \\
\mathbf{0} & 0
\end{array}\right]\left[\begin{array}{l}
\lambda \\
\mu
\end{array}\right]
\end{array}
$$

\footnotetext{
${ }^{3}$ Because $\operatorname{diag}\left\{\Delta^{*}\left(L^{-1}\right)\right\} \operatorname{diag}\left\{\Delta^{*} E\right\}$ is always invertible, the use of $\Delta^{*}$ instead of $\Delta$ in (10) ensure that $d \mapsto \lambda$ is a bijection.
} 
where the following notation have been introduced:

$$
E_{\delta}:=\operatorname{diag}\left\{\Delta^{*} E\right\}, \quad L_{\delta}:=\operatorname{diag}\left\{\Delta^{*}\left(L^{-1}\right)\right\}^{-1} .
$$

Eq. (12) reveals that if dynamics of both $v$ and $\sigma$ are coupled, they are completely disconnected from current distribution dynamics, related to $\delta$ trajectories. Also note inputs that $\mu$ and $\lambda$ can be used to independently control $(\sigma, v)$ and $\delta$, respectively. Those last two remarks have major consequences on control design (see Section V) and has to be regarded as the main result of [5].

\section{B. State-space decomposition}

From Section III, computation of a basis of $\mathscr{V}^{*}$ gives a way to isolate the largest region of the state-space which can be made both invariant and invisible from the output $z=v$, by means of a linear state feedback.

Since system (2) is controllable, $\mathscr{V}^{*}$ coincides with $\mathscr{R}^{*}$. Several techniques (see for instance [6, Sec. 4.3], [9] or [10]) allow us to compute a basis of $\mathscr{R}^{*}$. By means of one of them, we can prove that

$$
\mathscr{R}^{*}=\operatorname{Im}\left\{\left[\begin{array}{c}
\Gamma_{m} \\
\mathbf{0}_{m}^{\top}
\end{array}\right]\right\}
$$

where $\Gamma_{m} \in \mathbb{R}^{m \times(m-1)}$ is defined in (8). Note that $\mathscr{R}^{*}$ is a subspace of dimension $m-1$ since $\Gamma_{m}$ is full column rank.

We complete the state-space by introducing the following subspace $^{4}$ of $\mathbb{R}^{n}$ :

$$
\mathbb{R}^{n} / \mathscr{R}^{*}=\operatorname{Im}\left\{\left[\begin{array}{cc}
\mathbf{1}_{m} & \mathbf{0}_{m} \\
0 & 1
\end{array}\right]\right\} .
$$

since $\left[\Gamma_{m}, \mathbf{1}_{m}\right]$ is invertible as $\operatorname{Im}\left\{\mathbf{1}_{m}\right\}=\operatorname{Ker}\left\{\Gamma_{m}^{\top}\right\}$.

Concatenation of the two basis matrices above gives rise to a transformation matrix of the form

$$
T_{1}:=\left[\begin{array}{ccc}
\Gamma_{m} & \mathbf{1}_{m} & 0 \\
0 & 0 & 1
\end{array}\right] .
$$

whose first $m-1$ columns span $\mathscr{R}^{*}$, whereas the remaining part of $T_{1}$ complete the state-space. It comes out that matrix $T$ given by (9) is nothing but $T_{1} T_{2}$ where block diagonal $T_{2}$, defined as

$$
T_{2}:=\operatorname{diag}\left\{\left(\Gamma_{m}^{\top} \Gamma_{m}\right)^{-1},\left[\begin{array}{cc}
\frac{1}{m} & 0 \\
0 & 1
\end{array}\right]\right\},
$$

simply renormalizes $T_{1}$ without mixing up basis vectors of $\mathscr{R}^{*}$ and $\mathbb{R}^{n} / \mathscr{R}^{*}$ in this matrix, i.e. preserving confinement of basis vector $\mathscr{R}^{*}$ into the first $m-1$ columns of $T_{1} T_{2}$. Note that it is not necessary to add the matrix $T_{2}$ because its only purpose is to facilite the notations.

This discussion allows to interpret $\delta$ as coordinates related to $\mathscr{R}^{*}=\mathscr{V}^{*}$ or, equivalently, coordinate of the largest part of closed-loop state space (with some linear state-feedback) which does not impact the output $z=v$. This observation is physically meaningful as coordinates of the remaining part of the state-space are $(\sigma, v)$, so that increasing dimension of $\mathscr{R}^{*}$ will necessarily affects those variables and, in turn, affects voltage trajectory.

\footnotetext{
${ }^{4} \mathrm{~A}$ convenient way to obtain the state-space completion is to construct the quotient space of $\mathbb{R}^{n}$ modulo $\mathscr{R}^{*}$ defined in [7, Sec. 0.5] and characterized by $\operatorname{dim} \mathscr{R}^{*}+\operatorname{dim} \mathbb{R}^{n} / \mathscr{R}^{*}=\operatorname{dim} \mathbb{R}^{n}$
}

\section{Input-space decomposition}

Let us now isolate part of the input space which affects $\mathscr{R}^{*}$ and, hence, does not impact voltage trajectory. To this end, it suffices to (back) propagates $\mathscr{R}^{*}$ into the input space by computing $B^{-1} \mathscr{R}^{*}:=\left\{u \in \mathbb{R}^{m} \mid B u \in \mathscr{R}^{*}\right\}$ as this set (which is actually a vector space) contains input vectors that induce state trajectories in $\mathscr{R}^{*}$. Here $B^{-1} \mathscr{R}^{*}$ is trivial because $\operatorname{dim} \operatorname{Ker}\{B\}=0$ and its expression reads

$$
B^{-1} \mathscr{R}^{*}=\operatorname{Im}\left\{\operatorname{diag}\{E\}^{-1} \operatorname{diag}\{L\} \Gamma_{m}\right\} .
$$

To complete the input-space, we construct the following quotient space

$$
\mathbb{R}^{m} / B^{-1} \mathscr{R}^{*}=\operatorname{Im}\left\{\operatorname{diag}\{E\}^{-1} \operatorname{diag}\{L\} \mathbf{1}_{m}\right\}
$$

where $\operatorname{diag}\left\{E^{-1}\right\} \operatorname{diag}\{L\}$ has been incorporating into this expression as a way to simplify resulting transformation matrix of the input-space:

$$
G_{1}:=\operatorname{diag}\{E\}^{-1} \operatorname{diag}\{L\}\left[\begin{array}{ll}
\Gamma_{m} & \mathbf{1}_{m}
\end{array}\right] .
$$

As for the decomposition of the state space, change of coordinate of the input space provided in [5] and given by (10) can be recovered by normalizing $G_{1}$ via block diagonal matrix $G_{2}$ defined as

$$
G_{2}:=\operatorname{diag}\left\{\left(\Gamma_{m}^{\top} \Gamma_{m}\right)^{-1} E_{\delta} L_{\delta}^{-1}, \frac{E_{\mathrm{eq}}}{m L_{\mathrm{eq}}}\right\},
$$

so that $d=G\left[\lambda^{\top}, \mu\right]^{\top}$ with $G=G_{1} G_{2}$.

\section{Expression of the dynamics in the new coordinates}

Previous discussions can be sum up by expressing openloop (2) in the new coordinates:

$$
\left[\begin{array}{c:cc|c:c}
\mathbf{0} & \mathbf{0} & -\Delta\left(L^{-1}\right) & E_{\delta} L_{\delta}^{-1} & \mathbf{0} \\
\hdashline \mathbf{0} & 0 & -1 / L_{\mathrm{eq}} & \mathbf{0} & E_{\mathrm{eq}} / \bar{L}_{\mathrm{eq}} \\
\mathbf{0} & 1 / C & -1 /(R C) & \mathbf{0} & 0 \\
\hline \mathbf{0} & 0 & 1 & 0 & 0
\end{array}\right] .
$$

Observe now that (15) admits structure of (5) and, hence, its dynamics adopts cascaded form depicted by Fig. 2. This observation results from the fact system (2) enjoys the peculiar property that null matrix $\mathbf{0}$ is a friend of $\mathscr{R}^{*}$. Subsection III-B indeed predicts that (2) can be rewritten as in (5) but only as a closed-loop system and for some state-feedback $F \in \mathbb{R}^{m \times n}$. In fact, it comes out that, in the new coordinates, open-loop system (2) already admits the structure of (5) which means that null matrix $F=\mathbf{0}$ is already satisfactory for making $\mathscr{R}^{*}$ an output nulling invariant subspace.

The bottom line is the decomposition introduced in [5], i.e., $i$ and $d$ replaced by $(\delta, \sigma)$ and $(\lambda, \mu)$ respectively, follows from the construction of a basis of $\mathscr{R}^{*}$ and, in turn, of $B^{-1} \mathscr{R}^{*}$. 


\section{E. Trajectories $\delta(\cdot)$ corresponds to the internal dynamics}

Following the way (6) has been derived from (5), dynamics induced by (15) can be decomposed into a cascaded form where upper subsystem $\Sigma_{v}$ defined by

$$
\Sigma_{v}:\left\{\begin{aligned}
\frac{\mathrm{d}}{\mathrm{d} t}\left[\begin{array}{c}
\sigma \\
v
\end{array}\right] & =\left[\begin{array}{cc}
0 & -1 / L_{\mathrm{eq}} \\
1 / C & -1 /(R C)
\end{array}\right]\left[\begin{array}{c}
\sigma \\
v
\end{array}\right]+\left[\begin{array}{c}
E_{\mathrm{eq}} / L_{\mathrm{eq}} \\
0
\end{array}\right] \mu \\
z & =\left[\begin{array}{ll}
0 & 1
\end{array}\right]\left[\begin{array}{c}
\sigma \\
v
\end{array}\right]
\end{aligned}\right.
$$

feeds a lower one, denoted by $\Sigma_{\delta}$ and governs by

$$
\Sigma_{\delta}: \frac{\mathrm{d}}{\mathrm{d} t} \delta=-\Delta\left(L^{-1}\right) v+E_{\delta} L_{\delta}^{-1} \lambda
$$

Fig. 3 depicts the interactions between those two blocks.

From the discussion carried out in subsection III-B, $\Sigma_{v}$ corresponds to the external dynamic, i.e., the one which is visible from $z$, whereas $\Sigma_{\delta}$ is nothing but the internal dynamics which lies into the output nulling subspace $\mathscr{R}^{*}$ and, in turn, is invisible from $z$. Fig. 2 highlights this last comment by the fact the $\delta$ is a signal of the lower subsystem which does not affect the output $z=v$ of $\Sigma_{v}$, the higher block in the cascade. Property that internal dynamic does not impact the external one is thus recovered.

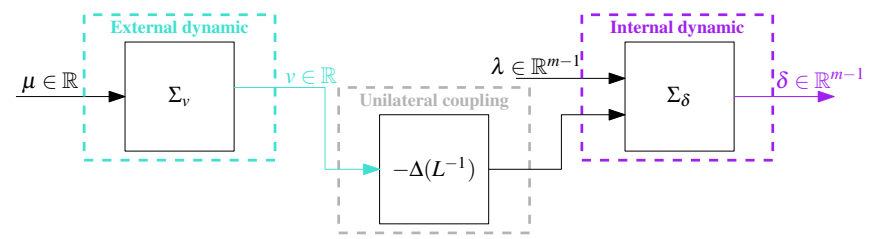

Fig. 3. New cascaded open loop model for buck interconnection

\section{CONTROL DESIGN}

The new formulation allows for modular design. Indeed, both voltage $v$ and current repartition $\delta$ can be controlled independently by the input $\mu$ and $\lambda$, respectively.

\section{A. External control design}

Interestingly, subsystem $\Sigma_{v}$ related to external dynamics corresponds to a single equivalent buck converter. As a result, we can apply any methodology from the literature to control it, including when $R$ is unknown. However, the purpose being to illustrate the paper results, we assume here that the load value $R$ is known for the sake of simplicity. Here, we choose to compute a state-feedback such that the external controller is expressed by:

$$
\mathscr{C}_{v}: \quad \mu=-K_{v}\left(\left[\begin{array}{c}
\sigma \\
v
\end{array}\right]-\left[\begin{array}{c}
v_{r} / R \\
v_{r}
\end{array}\right]\right)+\frac{v_{r}}{E_{\mathrm{eq}} R}
$$

where $K_{v} \in \mathbb{R}^{1 \times 2}$. This control law is nothing but a linear state-feedback stabilizing the shifted origin $(\sigma, v)-\left(v_{r} / R, v_{r}\right)$ and so that $v$ converges to $v_{r}$, provided that $K_{v}$ makes state matrix of closed-loop $\Sigma_{v}$ with $\mathscr{C}_{v}$ Hurwitz.

\section{B. Internal control design}

The considered controller is expressed by

$$
\mathscr{C}_{\delta}: \quad \lambda=-K_{\delta}\left(\delta_{r}-\delta\right)+L_{\delta} E_{\delta}^{-1} \Delta\left(L^{-1}\right) v .
$$

This control law gets rid of voltage influence on the current distribution by removing term $\Delta\left(L^{-1}\right) v$ so that derivatives of $\delta$ for closed-loop $\Sigma_{\delta}$ with $\mathscr{C}_{\delta}$ reads

$$
\dot{\delta}=-E_{\delta} L_{\delta}^{-1} K_{\delta}\left(\delta_{r}-\delta\right) .
$$

It follows that any matrix $K_{\delta} \in \mathbb{R}^{(m-1) \times(m-1)}$ making $-E_{\delta} L_{\delta}^{-1} K_{\delta}$ Hurwitz, ensures that $\delta$ converges to some prescribed current distribution vector $\delta_{r}$.

Note that, if voltage regulation is the unique control goal, $\mathscr{C}_{\delta}$ can actually be arbitrarily selected. However, controller (18) let the resulting closed-loop system enjoys internal asymptotic stability as an additional property. Furthermore, control law (18) can asymptotically drive $\delta$ to arbitrary reference $\delta_{r}$, so that not only voltage (and, in turn, total current) but also current distribution among converters are in hand. This gives a way to meet secondary objectives related to power-flow among the interconnection. Along this line, main result of [11] can be regarded as a control design achieving overall power losses minimization at the steadystate by means of appropriate selection of $\delta_{r}$.

From this discussion, characterization of $\mathscr{R}^{*}$ and $B^{-1} \mathscr{R}^{*}$ presented in this paper can also be viewed as a way to highlight degrees of freedom that remains in the control action once regulation of $z$ is achieved: If inputs belonging to $B^{-1} \mathscr{R}^{*}$ affect state trajectory, they do not impact regulated output and, hence, can be used to meet additional control goal. Important comments on this topic can be found in [12].

\section{Simulation Results}

In order to illustrate the state decomposition, simulations on the system (2) have been performed on Matlab/Simulink in the case of two converters. Note that using more than two converters do not add any difficulty for the control design.

1) Model parameters and reference definition: We consider two parallel converters $(m=2)$. Capacitor and resistor numerical values are respectively $C=47 \mu \mathrm{F}$ and $R=2 \Omega$. Other physical parameter values are listed by Table I. Voltage has to be regulated to $v_{r}=12 \mathrm{~V}$.

TABLE I

CONVERTERS PARAMETERS

\begin{tabular}{c|cc} 
& $k=1$ & $k=2$ \\
\hline$E_{k}(\mathrm{~V})$ & 20 & 25 \\
$L_{k}(\mathrm{mH})$ & 0.25 & 0.50
\end{tabular}

From previous discussions, full characterizing of the desired steady-state requires to define $\delta_{r}=i_{r 1}-i_{r 2} \in \mathbb{R}$, where $i_{r 1}$ and $i_{r 2}$ are current references of $i_{1}$ and $i_{2}$, respectively. The following two distinct scenarios are successively considered:

(i) $\delta_{r}=0 \Leftrightarrow i_{r 1}=i_{r 2}$ corresponding to uniform current distribution;

(ii) $\delta_{r}=-1 \Leftrightarrow i_{r 2}=i_{r 1}+1$ leading to steady-state for which converter 2 conveys more power than converter 1 . 
2) Simulation results: Following guidelines provided by Section V, controller gains are selected as follows:

$$
K_{v}=\left[\begin{array}{ll}
2 \times 10^{-3} & -36 \times 10^{-3}
\end{array}\right], \quad K_{\delta}=-5 .
$$

The two scenarios only differs on internal reference. As a result, external trajectories should be the same. This can be verified on Fig. 4 where voltage $v$ and total current $\sigma$ chronographs are identical for (i) and (ii). In fact, current repartition $\delta$, corresponding to $\mathscr{R}^{*}$ coordinates, is in the direction of $\left[\begin{array}{lll}1 & -1 & 0\end{array}\right]^{\top}$ which is contained in $\operatorname{Ker}\{C\}$ and, hence, invisible from $z$.
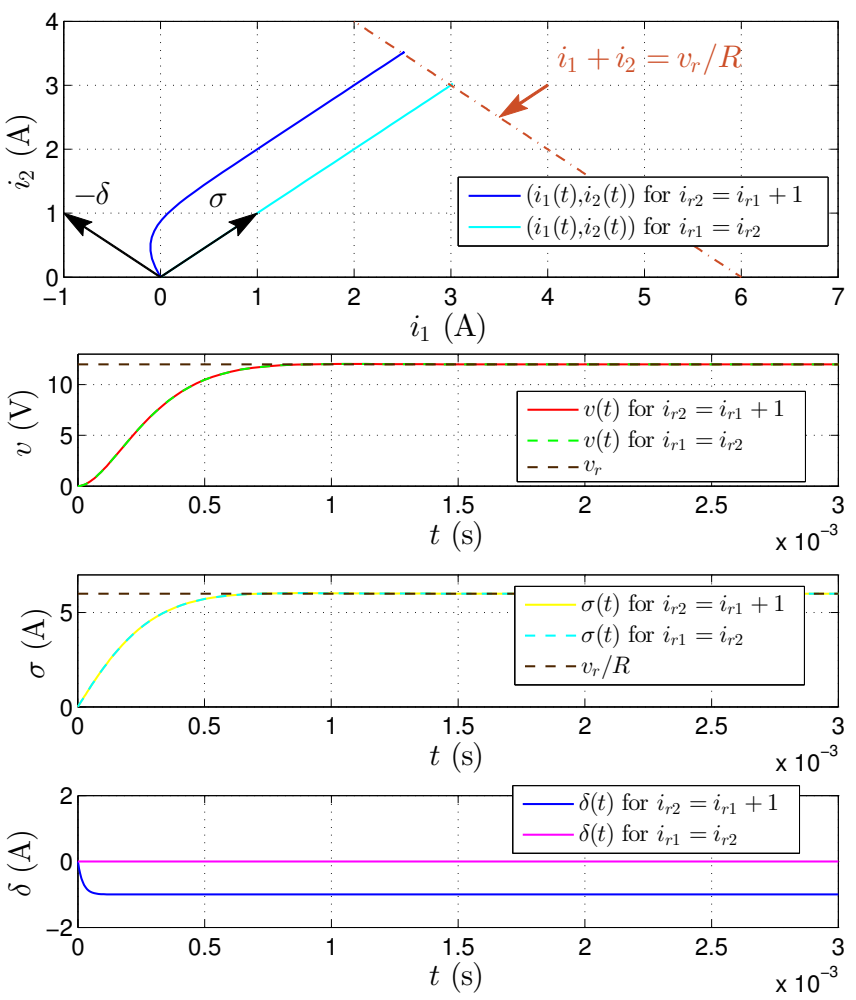

Fig. 4. Results in $\left(i_{1}, i_{2}\right)$ plan and chronographs of $v(t), \sigma(t)$ and $\delta(t)$

Figure 5 depicts closed-loop state trajectory for scenario (ii) in the state space $\left(i_{1}, i_{2}, v\right)$ using blue line. This trajectory is then decomposed into its external component (cyan line) lying in $\mathbb{R}^{n} / \mathscr{R}^{*}$ and its internal component (magenta line) which belongs to $\mathscr{R}^{*}$. To improve visual rendering, translation of $\mathscr{R}^{*}$ that contains equilibrium point is depicted (orange line) instead of $\mathscr{R}^{*}$ itself.

\section{CONCLUSION}

In this paper, a didactic methodology for separating voltage and current distribution dynamics has been proposed for parallel interconnection of buck converters. The core is the approach relied on characterization of controlled invariant subspaces contained in the kernel of the output map. This allows for change of both state and input coordinates giving rise to cascaded system for which voltage trajectory becomes independent from current distribution. Extensive discussion on benefits of this reformulation in terms of control design is also provided.

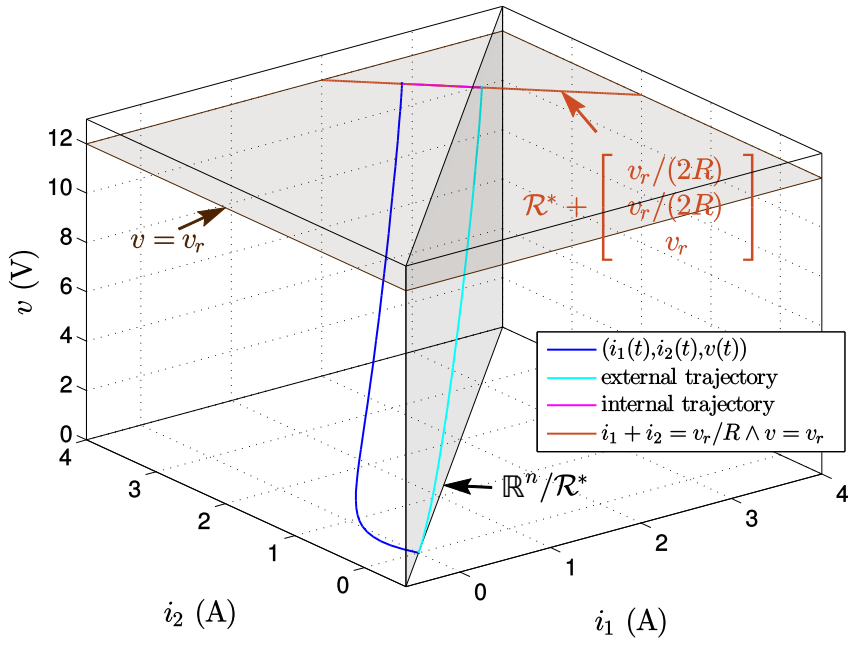

Fig. 5. Simulation results in $\left(i_{1}, i_{2}, v\right)$ plan for $\delta_{r}=-1 \mathrm{~A}$

\section{REFERENCES}

[1] J. M. Guerrero, J. C. Vsquez, and R. Teodorescu, "Hierarchical control of droop-controlled dc and ac microgrids ; a general approach towards standardization," in 2009 35th Annual Conference of IEEE Industrial Electronics, Nov 2009, pp. 4305-4310.

[2] V. J. Thottuvelil and G. C. Verghese, "Analysis and control design of paralleled $\mathrm{dc} / \mathrm{dc}$ converters with current sharing," in Proceedings of APEC 97 - Applied Power Electronics Conference, vol. 2, Feb 1997, pp. 638-646 vol.2.

[3] A. Cid-Pastor, R. Giral, J. Calvente, V. I. Utkin, and L. MartinezSalamero, "Interleaved converters based on sliding-mode control in a ring configuration," IEEE Transactions on Circuits and Systems I: Regular Papers, vol. 58, no. 10, pp. 2566-2577, Oct 2011.

[4] J. Sun, Y. Qiu, B. Lu, M. Xu, F. C. Lee, and W. C. Tipton, "Dynamic performance analysis of outer-loop current sharing control for paralleled dc-dc converters," in Twentieth Annual IEEE Applied Power Electronics Conference and Exposition, 2005. APEC 2005., vol. 2. IEEE, 2005, pp. 1346-1352.

[5] J.-F. Trégouët and R. Delpoux, "Parallel interconnection of buck converters revisited," IFAC-PapersOnLine, vol. 50, no. 1, pp. 15792 - 15797, July 9-14 2017.

[6] H. Trentelman, A. A. Stoorvogel, and M. Hautus, Control theory for linear systems, 2001st ed., ser. Communications and Control Engineering. Springer-Verlag London, 2012, vol. 1.

[7] W. M. Wonham, Linear multivariable control: a geometric approach. Springer-Verlag New York, 2012, vol. 10.

[8] G. Basile and G. Marro, Controlled and Conditioned Invariants in Linear System Theory/Book and Disk. Prentice Hall, 1992, vol. 1.

[9] G. Marro, F. Morbidi, L. Ntogramatzidis, and D. Prattichizzo, "Geometric control theory for linear systems: a tutorial," in Proceedings of the 19th International Symposium on Mathematical Theory of Networks and Systems MTNS 2010, July 2010, pp. 1579-1590.

[10] L. Ntogramatzidis and R. Schmid, "Robust eigenstructure assignment in geometric control theory," SIAM Journal on Control and Optimization, vol. 52, no. 2, pp. 960-986, 2014. [Online]. Available: https://doi.org/10.1137/130912906

[11] J.-F. Trégouët, R. Delpoux, and J.-Y. Gauthier, "Optimal secondary control for dc microgrids," in 2016 IEEE 25th International Symposium on Industrial Electronics (ISIE), Santa Clara, CA, June 2016, pp. 510-515.

[12] A. Serrani, "Output regulation for over-actuated linear systems via inverse model allocation," in 2012 IEEE 51st IEEE Conference on Decision and Control (CDC), Dec 2012, pp. 4871-4876. 